\title{
Study of the state a Francis turbine
}

\author{
Mariusz Żółtowski, Ph.D., \\ Bogdan Żółtowski, Prof., \\ University of Technology and Life Science in Bydgoszcz, Poland \\ Leonel Castaneda, Ph.D., \\ EAFIT University of Colombia
}

\begin{abstract}
This paper presents a methodology to evaluate the technical state of a Francis turbine by shaft rotor dynamic simulation. There are several rotor dynamic criteria that define the technical state of a turbo-machine. To feed the shaft rotor dynamic model this delivers the required information to accomplish the technical assessment. The numerical rotor dynamic model uses as input, the field forces obtained by the fluid-solid interaction analysis undertaken over the blades of the runner.

The rotor dynamic numerical simulations allow to determinate the record-in-time of the displacements of any point along the shaft. This information is relevant for diagnosis tasks, because it is possible to decompose it spectrally and to estimate the severity of the vibrations. Comparing the results of the numerical model against those obtained from machines that operates under normal conditions, it is possible to determinate the technical state of the turbo-machine. This allows studying the stability of the turbine working on several operation ranges.

A Francis turbine is a very complex machine that involves many physical phenomena of different nature. In this way, the hydraulic input forces needed by the rotor dynamic model should not be assumed but calculated directly from the fluid interaction over the turbine structure.
\end{abstract}

Key words: FEM; Francis turbine; diagnosis; fluctuations; hydrodynamic

\section{INTRODUCTION}

The technical diagnosis is a set organized of methods and a half that allow the evaluation of the technical condition state (his reason, evolution and consequence) of systems of engineering. In most cases they are systems in operation, designed to fulfill a principal function. These appliances generate and transform information, which is used later to study his technical condition state.

For the definition of the field of application of the technical diagnosis, are studied the processes of destruction of the objects during the whole period of his existence and certain situations, in which it is necessary to consider the actions (shares) of diagnosis. The term "period of existence of the machines" refers to the cycle that initiates in the instant that there are formulated the requirements of design that must fulfill the new object up to his respective liquidation (residues, felling and reutilization) (Żółtowski B., 2002).

The current technology (skill) is a result of the models' analysis, which they describe with certain degree of accuracy. The process, which takes as an aim the construction of the best model of operation (mathematical or empirical), is named a process of identification, and includes shaped, you try, estimation and check. The methodology of the studies of diagnosis includes the heuristic and mathematical models (Źółtowski B., 1996).

"The identification in the diagnosis is a process of searching complex, in that, based on the knowledge of the structure and the functioning of the object, there are realized the analysis and the synthesis of the model of the object for the obtaining of the information of diagnosis". The idea on the methodical construction of the dynamic model of the mechanical system is supported of the information of the modal experiment and the information of the measurements of the quantities of vibration (acceleration of the vibration, moment rotational, force and etc.) in conditions of exploitation development. The models of analysis of loads are constructed depending on his application in the procedures of dynamic optimization and diagnosis of exploitation development. (Źółtowski B., Cempel C., 2004).

During the electric generation process, the turbines develop hydraulic phenomena that extend along all the components of the machine represented in vibrations, sound and instabilities. Recently, the evaluation of the machine state using modern diagnosis techniques has become important. These diagnosis techniques, expert systems in particular, are based on the knowledge of the technical system and on the 
use of artificial intelligence. To enlarge the understanding of machines for failure detection, the study of their dynamical behavior plays a significant role. This methodology is known as maintenance based on models and pretends to achieve the failure identification by establishing relations between the real object and a virtual model (Kicinski, 2006).

The vibration analysis has been a important tool in the technical diagnosis. Instruments for measuring vibrations (accelerometers) are implemented in large machines and the parameters extracted from the acquired signals are used as effective indicators of the technical state. The rotor dynamical study of turbines, in this case, pretends to develop the knowledge to correctly identify the relations between the symptom and its causes.

The objective of the present study is to develop a study method that allows calculating the displacements of any point along the shaft of the turbine using FEM simulations. This information is relevant for diagnosis tasks, because it is possible to decompose it spectrally and to estimate the severity of the vibrations.

\section{CASE OF STUDY}

This investigation considers a Francis turbine composed by 20 stay and guide blades and 15 runner blades. It rotates at 900 rpm and generates a maximum output of 10MW. For this case, only the Best Efficiency Point (BEP) is analyzed. The turbine head is $230 \mathrm{~m}$ and the discharge at this point is $4.8 \mathrm{~m}^{3} / \mathrm{s}$.

The assembly of the turbine / generator can be observed in Fig. 1. The set is assembled on a rigid shaft, to which are attached: the runner, the flywheel, the generator core and poles, and the exciter. The shaft is supported by three hydrodynamic bearings. The first of them (nearest to the runner) has axial and radial effect; the other two have only radial effect.

\section{METHOD OF EXPERIMENTAL MEASURES}

A series of measurements using dynamic pressure sensors arranged along the guide vanes and draft tube were carried out. Also, a set of strain gages installed in the turbine shaft were employed with the purpose of measuring deformations to determine the torque and the radial forces.
There were installed sensors along different components of the turbine. Three of them were installed near the zone where the rotor-stator interaction occurs (between the guide vane and the runner). Fig. 2 shows an image of the approximate position of one sensor.

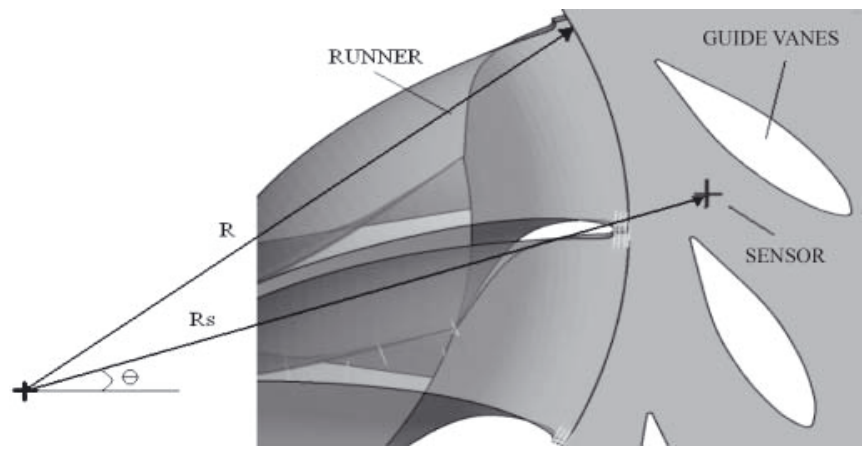

Fig. 2. Position of the sensors

The task of these sensors was to capture pressure fluctuations generated by the rotor-stator interactions. Fig. 3 shows an image of the experimental assembly carried out. It can be appreciated the physical conditions of the sensor installation.

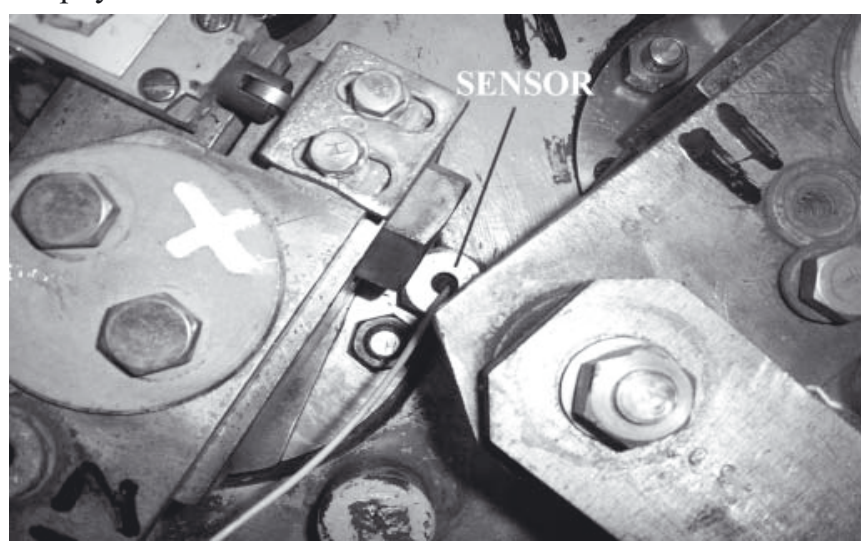

Fig. 3. Photo of the experimental location of the sensor

Another six dynamic pressure sensors were installed along the draft tube. The objective of the sensors was to obtain

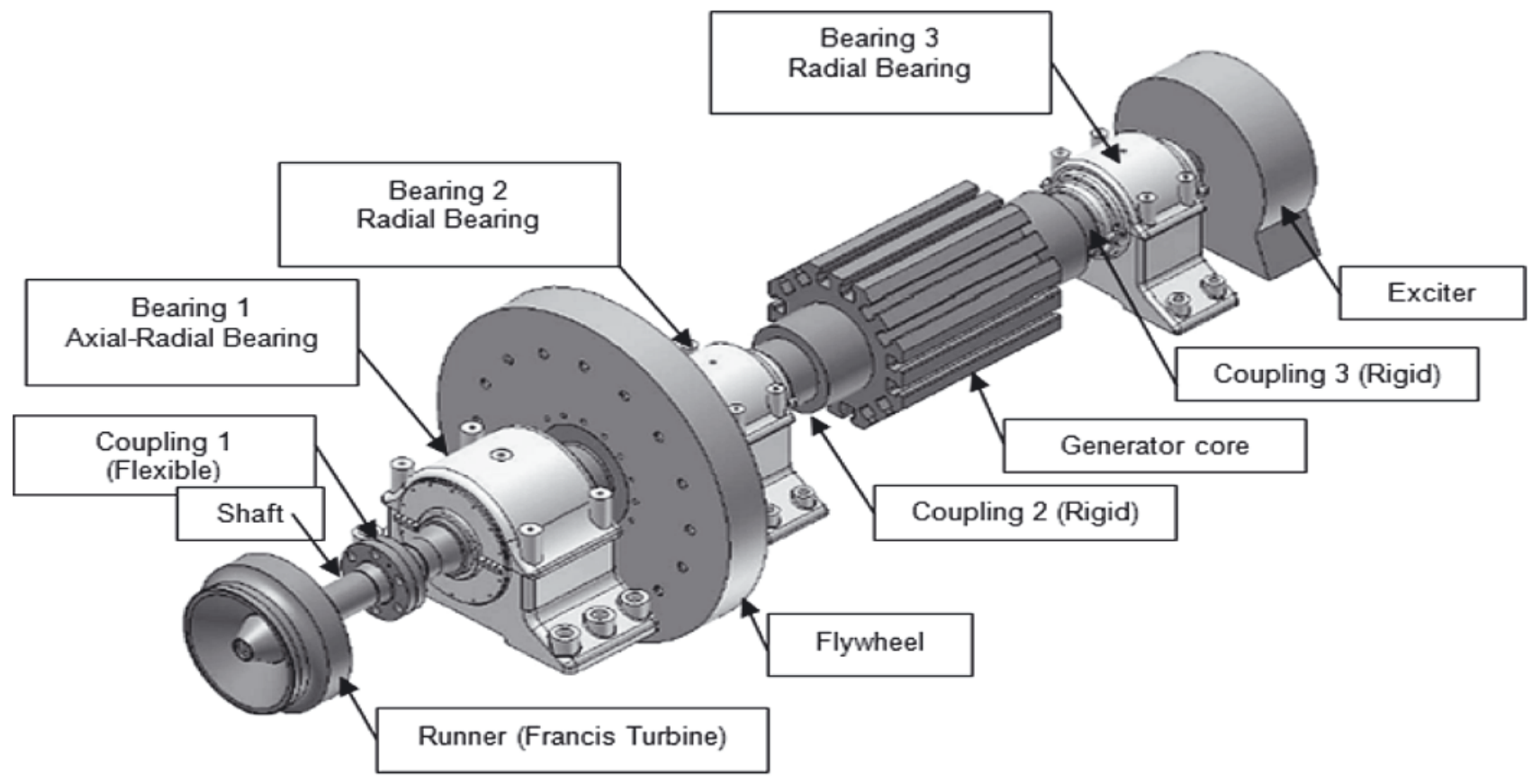

Fig. 1. Turbine shaft with bearings and attached mechanical elements 
information on the vortex rope generated at the exit of the runner. The sensors were distributed in two cross sections: i.e. $\mathrm{A}$ and $\mathrm{B}$; in each section were installed 3 sensors (Fig. 4).
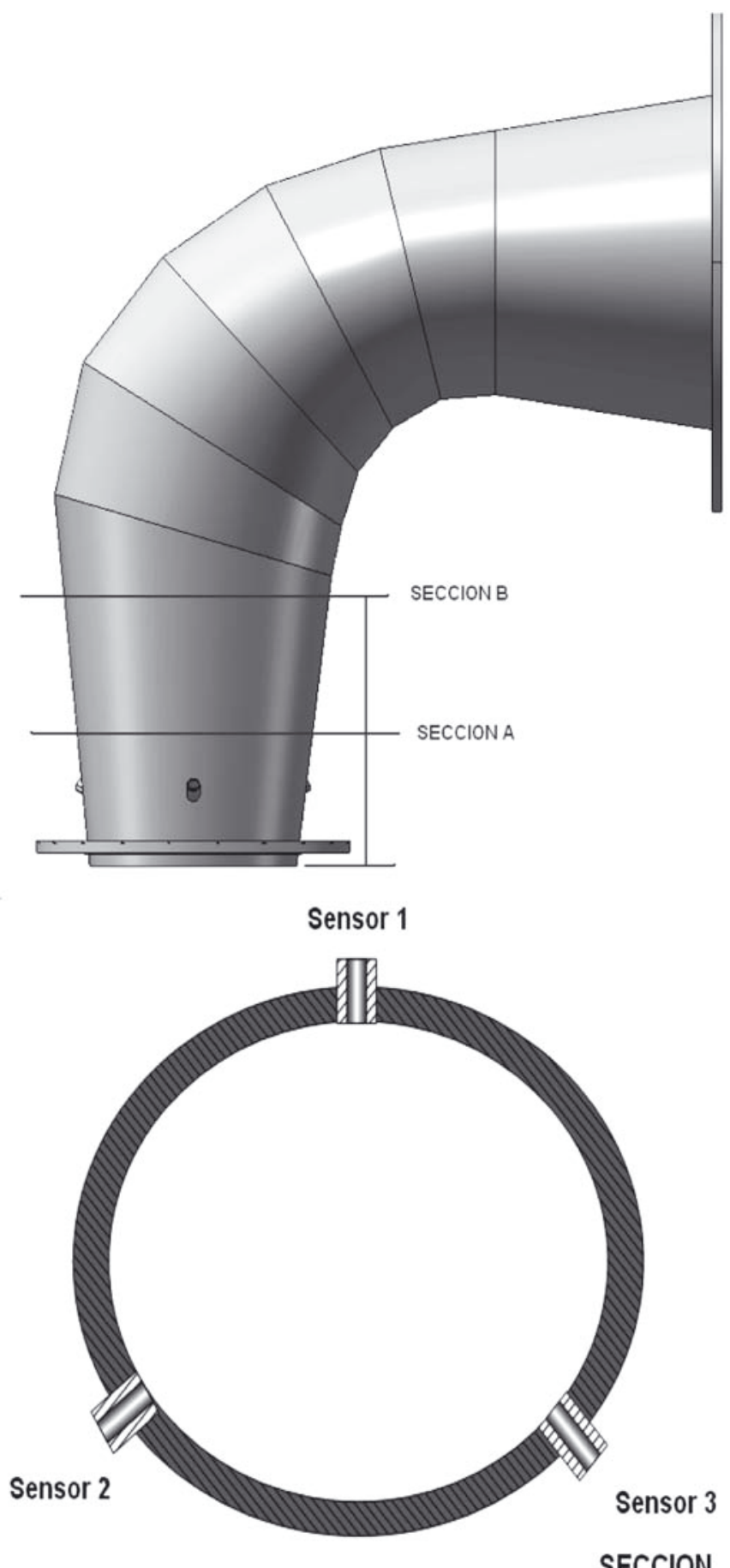

Fig. 4. Distribution of the sensors in the draft tube

The measurements were recorded performing a simultaneous sampling during 3 seconds of every channel at $102 \mathrm{kHz}$, i.e. more than 40 rotor revolution and more than 6500 samples per channel. It allows to capture all the hydraulic phenomena present at low and high frequencies.

For the measurement of the torque and the radial force a strain gage technique applied to the generation shaft was used. The use of this technique did not affect the turbine operation in any way. The strain gage signals were registered via wireless connection.

Fig. 5 shows the position in the shaft where the strain gages (SG) were installed. For the measurement of the radial force it was use of four bi axial well doable SG meanwhile the measurement of the torque used one bi axial well doable SG. The strain gages were connected to a transmitter tied to the shaft.

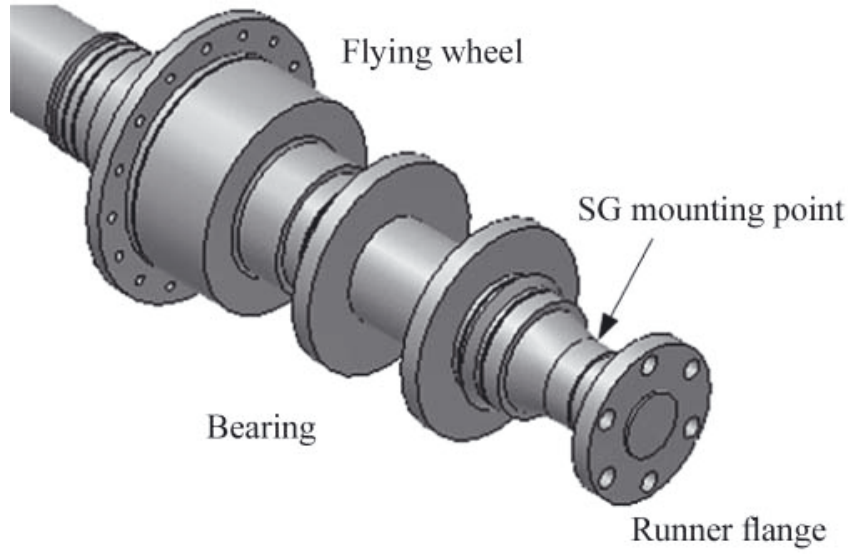

Fig. 5. Strain gages position in the shaft

Fig. 6 shows an image of the experimental installation of the SG. The distribution of the strain gages and its position in the shaft can be observed.

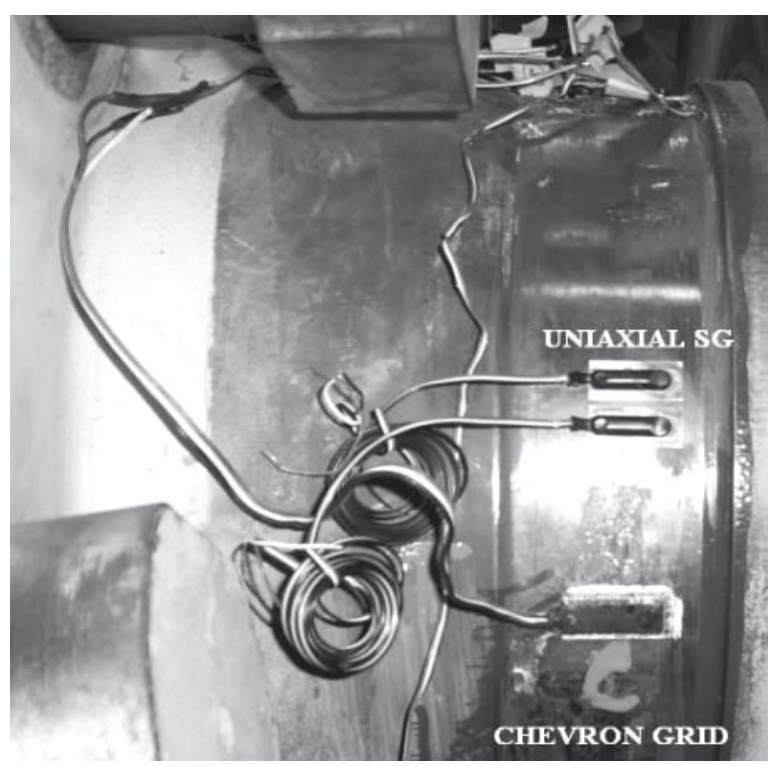

Fig. 6. Photo of the experimental installation of the gages in the shaft

The information give by all this pressure sensors and the strain gages is used to validate the CFD numerical model.

\section{ROTODYNAMIC METHOD}

The goal is to develop a model that accurately predicts the dynamic behavior of the shaft of a Francis turbine by means of numerical methods. To achieve this goal, it is necessary to find an approximated solution to the equations of motion of the system. These equations are a set of non linear differential equations:

$$
\mathbf{M} \ddot{\mathbf{x}}+\mathbf{D}(\mathbf{x}, \dot{\mathbf{x}}) \dot{\mathbf{x}}+\mathbf{K}(\mathbf{x}, \dot{\mathbf{x}}) \mathbf{x}=\mathbf{P}(\mathbf{t})
$$

where:

M - global matrix of inertia

D - global matrix of damping

$\mathrm{K}$ - global matrix of stiffness

$\mathrm{P}(\mathrm{t})$ - generalized vector of external excitations

$\mathrm{x}-$ generalized vector of position 
The model is implemented in the computational environment MESWIR ${ }^{\circledR}$. This software was developed by the Institute of Fluid Flow Machinery (IFFM) from the Polish Academy of Science. It composed by a set of codes to determine static and dynamic parameters of rotors, including analysis of the hydrodynamic bearings. The software uses the finite element method to analyze the rotor shaft and the finite difference method to determine the characteristic of the hydrodynamic bearings. With the software it is possible to analyze lateral vibrations with linear and non linear models.

The required entries of the model are: the conditions of support, the forces or external excitations, the mechanical and geometrical properties of the shaft. The shaft is supported in three hydrodynamic bearings but for the rotordynamic model they are represented by springs and dampers. The determination of the oil film stiffness and damping coefficients required additional computation of the fluid dynamics involved. The oil film stiffness and damping coefficients depend on the relative position and velocity of the journal in the bearing causing the non linearity in the equations of motion. However the results obtained under the assumption of linear stiffness and damping coefficients are acceptable. For the purpose of this work, the MESWIR ${ }^{\circledR}$ linear models were used.

The bearings are also attached to a foundation. The foundation is replaced in the mechanical model by springs and dampers, whose coefficients were prior determined by experimental measurements and numerical simulations. The shaft is modeled coupled rigid discs with masses and inertias equivalent to those of the real objects.

\section{Geometry and physical properties}

The real model is simplified in a mechanical model to make possible its analysis (Fig. 7). The shaft is discredited into 33 cylindrical Timoshenko beam elements with six degrees of freedom to use the finite element method. These elements are provided of mass, stiffness and damping, which are estimated from the physical properties of the material of the shaft, such as: density, Young modulus, Poisson coefficient and damping coefficients. In the Tab. 1 are listed the material properties.
Tab. 1. Physical properties of the material of the shaft.

\begin{tabular}{|c|c|c|c|c|}
\hline Density & $\begin{array}{c}\text { Young } \\
\text { modelus }\end{array}$ & $\begin{array}{c}\text { Poison } \\
\text { coefficient }\end{array}$ & $\begin{array}{c}\text { Damping } \\
\text { coefficient A }\end{array}$ & $\begin{array}{c}\text { Damping } \\
\text { coefficient } \\
\boldsymbol{\beta}\end{array}$ \\
\hline $\begin{array}{c}7800 \\
\mathrm{~kg} / \mathrm{m}^{3}\end{array}$ & $200 \mathrm{GPa}$ & 0.3 & $1.96 \mathrm{rad} / \mathrm{s}$ & $\begin{array}{c}5.09 * 10^{-5} \\
\mathrm{~s} / \mathrm{rad}\end{array}$ \\
\hline
\end{tabular}

\section{Support parameters}

In the oil film in the hydrodynamic bearings appears a hydrodynamic pressure distribution induced by the rotation and originating the reaction force. This pressure distribution is described by the Reynolds equation:

$$
\frac{1}{\mathrm{R}^{2}} \frac{\partial}{\partial \theta}\left(\mathrm{h}^{3} \frac{\partial \mathrm{p}}{\partial \theta}\right)+\frac{\partial}{\partial \mathrm{z}}\left(\mathrm{h}^{3} \frac{\partial \mathrm{p}}{\partial \mathrm{z}}\right)=6 \eta\left(\frac{1}{\mathrm{R}} \frac{\partial(\mathrm{hU})}{\partial \theta}+2 \frac{\partial \mathrm{h}}{\partial \mathrm{t}}\right)
$$

where:

$\mathrm{p}$ - pressure in the oil film

$\mathrm{h}$ - film thickness

$\mathrm{U}$ - oil velocity in tangential direction

$\eta-$ dynamic oil viscosity

$\mathrm{R}$ - radius of journal

Assuming that the flux is laminar and that the viscosity is constant along the oil film the pressure distribution could be found. The magnitude and orientation of the reaction force depend of the position and velocity of the gravity center of the rotor shaft. The stiffness and damping coefficients are computed based on the reaction force. The stiffness coefficients are the derivatives of the reaction force respect to the displacements.

$$
\begin{array}{ll}
\mathrm{k}_{11}=\frac{\partial \mathrm{W}_{\mathrm{x}}}{\partial \mathrm{x}}, & \mathrm{k}_{22}=\frac{\partial \mathrm{W}_{\mathrm{y}}}{\partial \mathrm{y}} \\
\mathrm{k}_{12}=\frac{\partial \mathrm{W}_{\mathrm{x}}}{\partial \mathrm{y}}, & \mathrm{k}_{22}=\frac{\partial \mathrm{W}_{\mathrm{y}}}{\partial \mathrm{x}}
\end{array}
$$

where:

$\mathrm{k}_{11}, \mathrm{k}_{22}, \mathrm{k}_{12}, \mathrm{k}_{22}$ - oil film stiffness coefficients

$\mathrm{W}_{\mathrm{x}}, \mathrm{W}_{\mathrm{y}} \quad-$ components of the reaction force

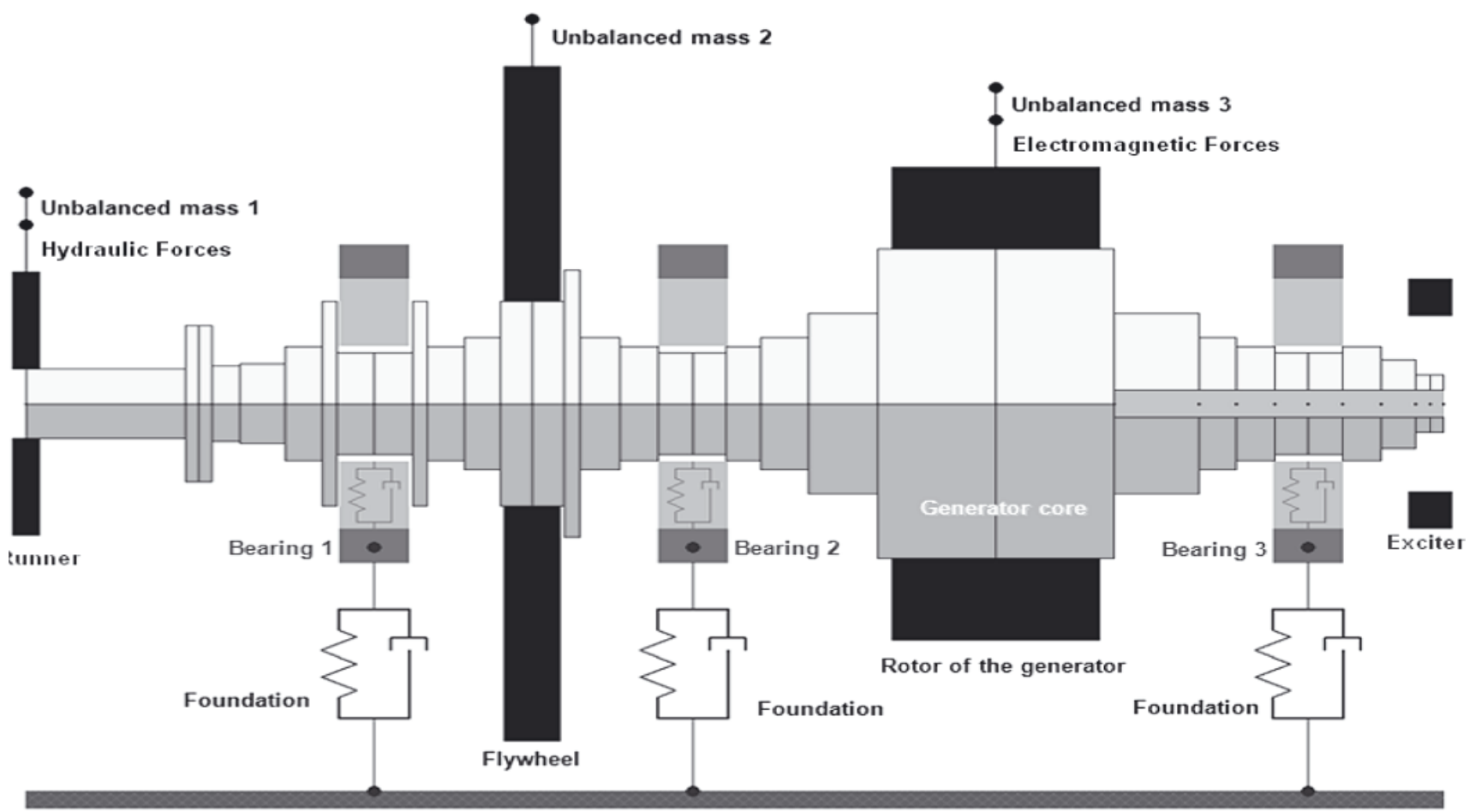

Fig. 7. Numerical model 
The damping coefficients are found as the derivatives of the reaction force respect to the velocities.

$$
\begin{array}{ll}
c_{11}=\frac{\partial W_{x}}{\partial \dot{x}}, & c_{22}=\frac{\partial W_{y}}{\partial \dot{y}} \\
c_{12}=\frac{\partial W_{x}}{\partial \dot{y}}, & c_{22}=\frac{\partial W_{y}}{\partial \dot{x}}
\end{array}
$$

where:

$\mathrm{c}_{11}, \mathrm{c}_{22}, \mathrm{c}_{12}, \mathrm{c}_{22}-$ oil film damping coefficients

The Fig. 8 presents a representation of the oil film as springs and dampers.

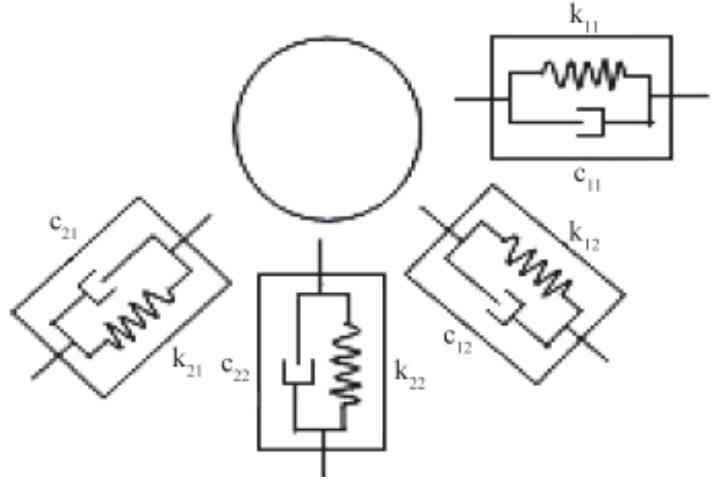

Fig. 8. Stiffness and damping coefficients the oil film

The shaft is affected by static and dynamic loads. The static loads are the masses of the elements attached to the shaft. The dynamic loads are originated by: residual unbalanced masses, the solid-fluid interaction in the runner and electromagnetic phenomena in the generator.

\section{Static and dynamic loads}

The static loads are determined by the geometry and physical properties of the discs assembled to the shaft. For the model, it is supposed that these discs are made of steel. The discs add mass and inertia to the system but they do not represent additional stiffness or damping. Other properties of the discs are list in Tab. 2.

Tab. 2. Elements assembled to the shaft

\begin{tabular}{|c|c|c|c|c|}
\hline Element & Runner & Flywheel & $\begin{array}{c}\text { Generator } \\
\text { poles }\end{array}$ & Exciter \\
\hline $\begin{array}{c}\text { Outer } \\
\text { diameter [m] }\end{array}$ & 0.900 & 2.300 & 1.614 & 0.850 \\
\hline $\begin{array}{c}\text { Inner diameter } \\
{[\mathrm{m}]}\end{array}$ & 0.240 & 0.706 & 1.054 & 0.600 \\
\hline $\begin{array}{c}\text { Thickness } \\
{[\mathrm{m}]}\end{array}$ & 0.146 & 0.300 & 1.090 & 0.225 \\
\hline $\begin{array}{c}\text { Mass } \\
{[\mathrm{kg}]}\end{array}$ & 673 & 8806 & 9977 & 500 \\
\hline $\begin{array}{c}\text { Inertia } \\
{\left[\mathrm{kgm}^{2}\right]}\end{array}$ & 73 & 6372 & 4634 & 68 \\
\hline
\end{tabular}

As it was mentioned the dynamic loads could be inertial, hydraulic or magnetic. The inertial forces are used to tune the model and the represent an output of the model. The hydraulic forces are estimated by CFD simulations. Regarding to the magnetic forces only unbalanced magnetic pull is considered.

The unbalanced magnetic pull appears when the rotor is eccentrically positioned respect to the stator. This causes asymmetric distribution of the magnetic flux and then an unbalance force in the direction of the smallest air gap. This force depends in a non linear way of the relative eccentricity, which is defined as:

$$
\varepsilon=\mathrm{u}_{\mathrm{r}} / \Delta \mathrm{R}
$$

where:

$\varepsilon \quad-$ relative eccentricity

$\mathrm{u}_{\mathrm{r}} \quad-$ distance between the rotor center line and the stator center line

$\Delta \mathrm{R}$ - difference between the stator inner radius $\left(\mathrm{R}_{\mathrm{s}}\right)$ and the rotor radius $\left(R_{r}\right)$

The unbalance magnetic pull has a constant and a variable part. The variable force oscillates with a frequency equal to the supply frequency. If the number of poles increases, the variable part decreases. The mean value for the unbalance magnetic pull could be found as (Gustavsson, 2005):

$$
\mathrm{F}=\frac{\mu_{0} \mathrm{~S}_{\mathrm{S}}^{2} \mathrm{R}_{\mathrm{s}}^{3} \mathrm{~h} \pi}{2 \mathrm{p}^{2} \Delta \mathrm{R}^{2}} \frac{\mathrm{e}}{\sqrt{\left(1-\mathrm{e}^{2}\right)^{3}}}
$$

where:

$\mu_{0}-$ magnetic permeability

$\mathrm{S}_{\mathrm{s}}-$ lineal density of current

$\mathrm{R}_{\mathrm{s}}-$ stator inner radius

$\mathrm{h}$ - rotor length

e - relative eccentricity

$\mathrm{p}-\mathrm{nNumber}$ of poles

By means of a static analysis the deflection of the shaft in the generator core section is found. With this value and the technical information of the machine the unbalance magnetic pull is computed and is found that it has a value of $4966 \mathrm{~N}$.

\section{RESULTS}

The experimental data used in rotor dynamic calculations is obtained from displacement sensors installed in the bearings. In each bearing are two sensors forming a rectangular angle between them and an angle of 45 degrees respect the horizontal plane as is shown in Fig. 9. Then, the vibration is measure in a coordinate system that is 45 degrees rotated respect the general coordinate system and some transformations should be made to compare the results.

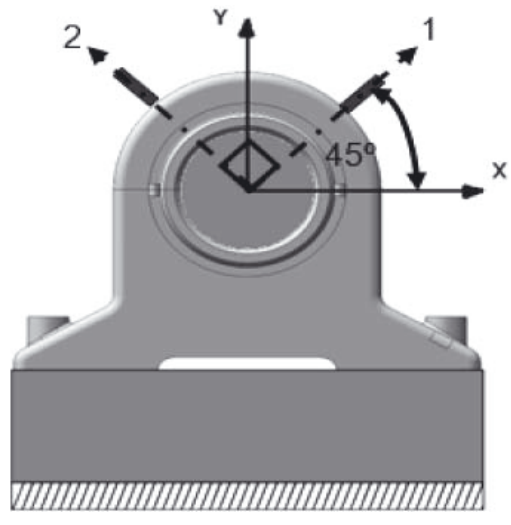

Fig. 9. Place of the sensors on the rotor

The most relevant component in the spectrum is at $15 \mathrm{~Hz}$, which is the rotational frequency of the machine. The other force components are very low and are not considered in this analysis. In the model the force should be introduced as an unbalanced mass at specific unbalance radius. The transformation is made using the definition of centrifugal acceleration. 


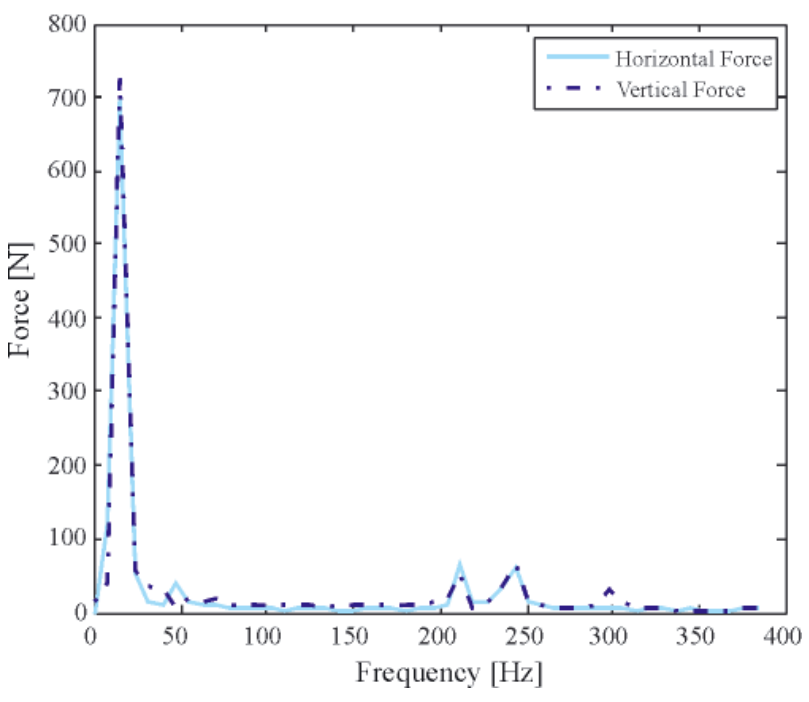

Fig. 10. Spectral analysis of the radial force

Tab. 3. Spectral components of the radial force.

\begin{tabular}{|c|c|c|}
\hline $\begin{array}{c}\text { Frequency } \\
{[\mathbf{H z}]}\end{array}$ & $\begin{array}{c}\text { Horizontal force } \\
{[\mathbf{N}]}\end{array}$ & $\begin{array}{c}\text { Vertical force } \\
{[\mathbf{N}]}\end{array}$ \\
\hline 15 & 696.2 & 723.50 \\
\hline 210 & 65.53 & 53.52 \\
\hline 240 & 61.58 & 64.93 \\
\hline 300 & - & 30.60 \\
\hline
\end{tabular}
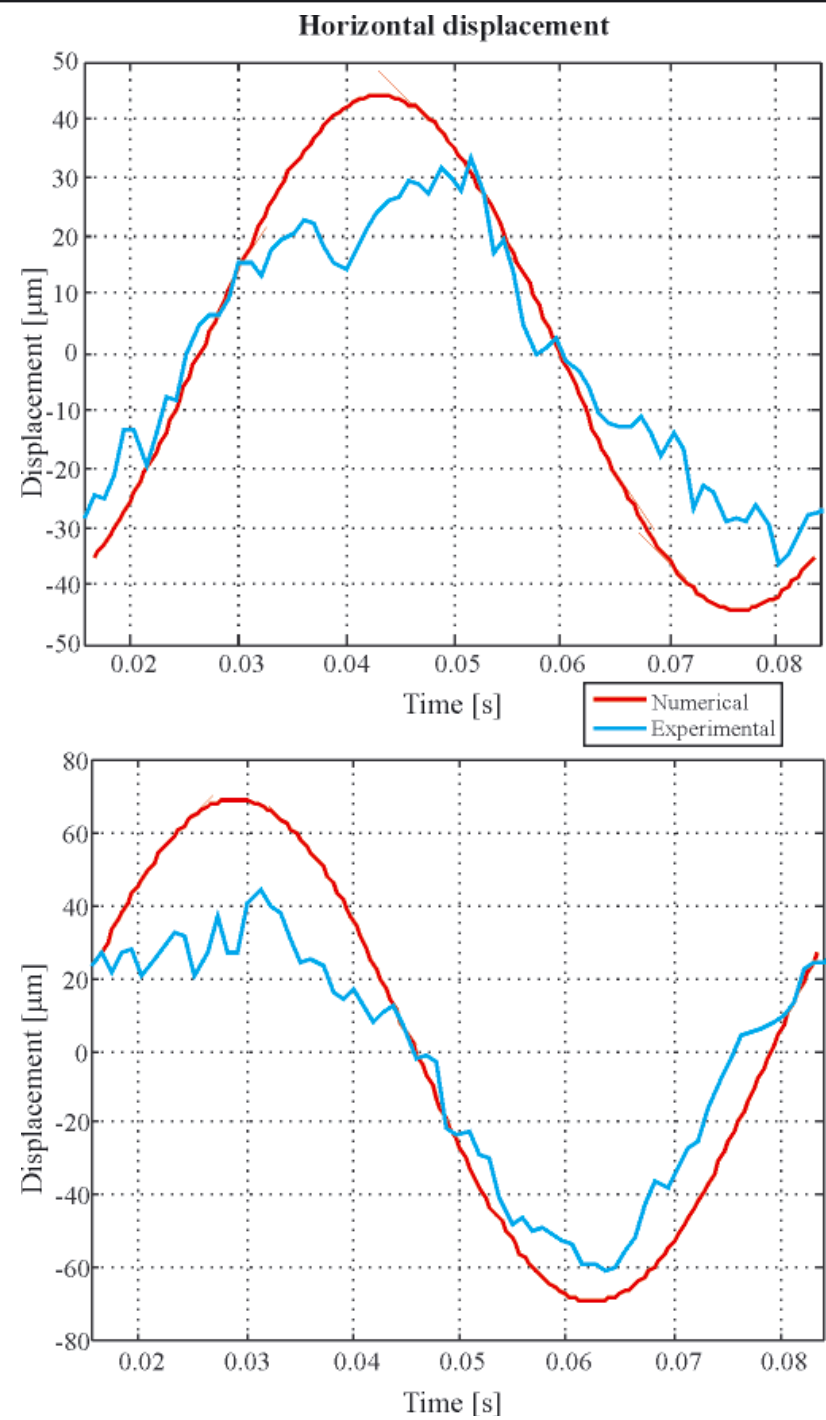

Fig. 11. Numerical and experimental displacement signals for the bearing 1
The analyses are made with the modules of MESWIR ${ }^{\circledR}$ for studying lateral vibrations with linear models. Hence, only excitations at first harmonic are taken into account and the numerical signals are sine functions with the rotational frequency. In Fig. 11, 12 and 13 the experimental and numerical results for the displacement vibration are compared for each bearing.

Horizontal displacement
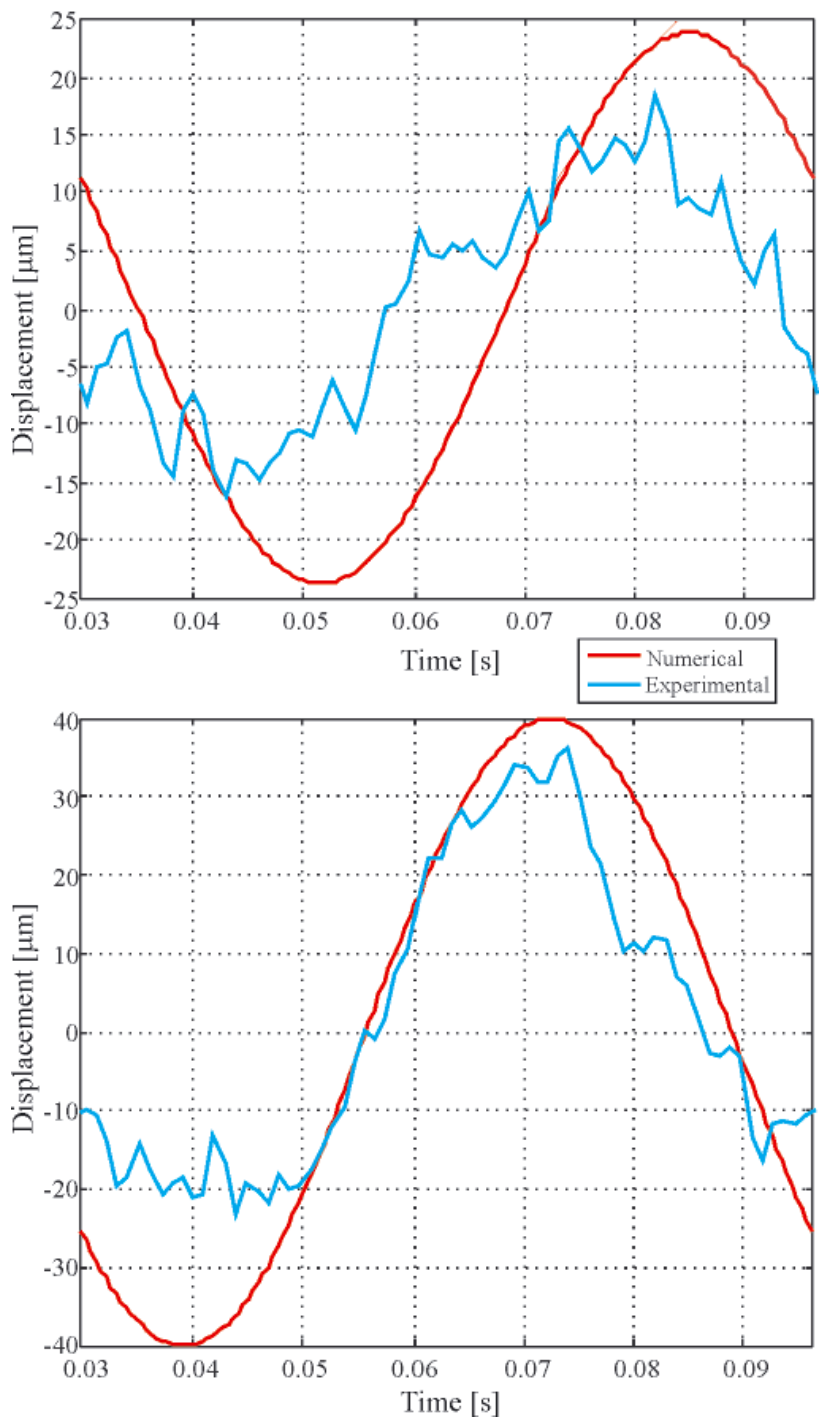

Fig. 12. Numerical and experimental displacement signals for the bearing 2

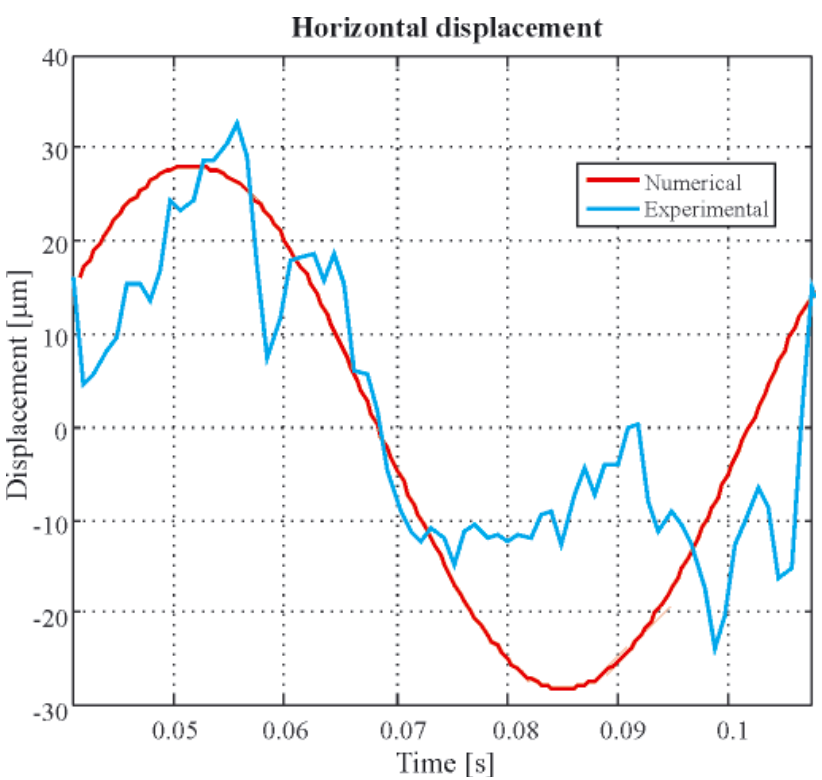

Fig. 13. Numerical and experimental displacement signals for the bearing 3 
As it was mentioned the unbalanced forces are tuned until the numerical vibration amplitudes adjust to those measured in the real technical object. The final required values represent a result from the rotor dynamic model.

Tab. 4. Resultant unbalance masses

\begin{tabular}{|c|c|c|c|}
\hline Position & $\begin{array}{c}\text { Unbalance } \\
\mathbf{d} \text { mass } \\
{[\mathbf{k g}]}\end{array}$ & $\begin{array}{c}\text { Unbalance } \\
\text { e radius } \\
{[\mathbf{m}]}\end{array}$ & $\begin{array}{c}\text { Phase } \\
\text { [degrees] }\end{array}$ \\
\hline Runner & 7.000 & 0.5 & 0 \\
\hline Flywheel & 15.000 & 0.5 & 180 \\
\hline Generator poles & 12.400 & 0.5 & 0 \\
\hline $\begin{array}{c}\text { Runner } \\
\text { (hydraulic force) }\end{array}$ & 0.157 & 0.5 & 0 \\
\hline $\begin{array}{c}\text { Runner } \\
\text { (hydraulic force) }\end{array}$ & 0.163 & 0.5 & 90 \\
\hline
\end{tabular}

\section{FINAL CONSIDERATIONS}

The radial forces obtained via numerical methods were used to feed the FEM rotor dynamic software. These forces have a consistent predominant spectrum component at the frequency of rotation which was used in the linear rotor dynamic model. However this resultant radial component has a low magnitude, because for the simulations the runner is completely symmetrical and the unbalance in the pressure distribution is little. If some imperfections of the runner are included in the simulations, the magnitude of force would probably become more significant.

It was possible to develop a rotor dynamic model whose results are in agreement with the experimental data. In this case, the unbalance masses required are relative large. This could be caused because the rotor is very rigid and important amounts of residual unbalanced could be bear with acceptable vibration amplitudes.

The amplitudes of vibration for the displacement obtained by the numerical computations and the experimental measures are acceptable according to the standard ISO 7919-5. This standard establishes a maximum limit of $165 \mu \mathrm{m}$. From the point of view of vibration the technical state of the machine is acceptable.

\section{BIBLIOGRAPHY}

1. Egusquiza E., Nascimento L.P., Valero, C. Jou, E. (1994). El diagnostico de dańos en grupos hidroeléctricos mediante el análisis de vibraciones. Revista Ing. del agua Vol 1 Num 3.

2. Gagnon J.M., Payette F.A. and Deschenes C. (2007). Numerical Simulation of a Rotor-Stator Unsteady Interaction in a Propeller Turbine. CFD Society of Canada, Toronto.

3. Guedes A., Kueny J.L., Ciocan G. and Avellan F. (2002). Unsteady rotor-stator analysis of a hydraulic pump-turbine - CFD and experimental approach.

4. Proceedings of the XXIst IAHR symposium on hydraulic machinery and systems, Laussane, Switerland.
5. Hoffmann K. (1989). An introduction to measurements using strain gages. Hottinger Baldwin Messtechnik GmbH, Darmstadt.

6. Menter F.R. (1994). Two - equation eddy viscosity turbulence models for engineering applications. AIAA Journal.

7. Nennemann B. and Farhat M. (2005). CFD prediction of unsteady wicket gate-impeller interaction in Francis turbines: A new standard hydraulic design procedure. Waterpower XIV, Austin, USA

8. Qian Z., Yang J., Huai W. (2007). Numerical simulation and analysis of pressure fluctuation in Francis hydraulic turbina with air admisión. Journal of hydrodynamics.

9. Zobeiri A., Kueny J.L., Farhat M. and Avellan F. (2006). Pump turbine rotor-stator interactions in generating mode: pressure fluctuations in distributor channel. 23th IAHR symposiums, Yokohama, Japan.

10.Ciocan and others (2007). Experimental study and numerical simulation of the FLINDT draft tube rotating vortex. Journal of fluid dynamics. Vol 129.

11.Kicinski J. (2006). Rotor dynamics. Institute of Fluid-Flow Machinery Polish Academy of Science, Gdansk.

12.Gustavsson R. (2005). Modelling and Analysis of Hydropower Generator Rotors. Lulea University of Technology. The Polhem Laboratory. Division of Computer Aided Design.

13.Zoltowski B., Castaneda Heredia LF. (2009). Estudio de explotación de vehiculos ferroviarios. EAFIT University, Colombia, pp.298.

14.Zoltowski B., Castaneda Heredia LF. (2009). Research rail vehicles. Transportation. UTP, Bydgoszcz, pp.220.

15.Zoltowski B.(2011). Fundamentals of diagnostic machines. UTP Bydgoszcz, pp.200.

16.Zoltowski B., Castaneda Heredia LF. (2010). Bases del diagnostico tecnico de maquinas. EAFIT University, Colombia, pp.233.

17.Zoltowski B., Lukasiewicz M. (2012). Diagnosis of Vibration machines. ITE-PIB, Radom.

18.Zoltowski B., Landowski, B., Przybyliński B. (2012). Designing the operation of machinery. UTP, Bydgoszcz.

19.Zoltowski, B., Lukasiewicz M., Kałaczyński T. (2012). Information technology in the study of machines. UTP, Bydgoszcz.

20.Zoltowski B. (2012). Methods of virtual engineering in clinical status, security threats operated machines and the environment. UTP, Bydgoszcz.

21.Zoltowski M. (2012) Operational modal analysis study design building. UTP, Bydgoszcz.

\section{CONTACT WITH THE AUTHORS}

Mariusz Żółtowski, Ph.D., Bogdan Żółtowski, Prof.,

University of Technology and Life Science in Bydgoszcz,

Ks. Kordeckiego 20, 85-225 Bydgoszcz, POLAND

mobile: +48791626848

mazolto@utp.edu.pl bogzol@utp.edu.pl

Leonel Castaneda, Ph.D.,

EAFIT University of Colombia

Cr 49 N7 s 50 Medellín, COLOMBIA 